\title{
La cour de récréation au prisme du genre, lieu de transformation des responsabilités des enseignant-e-s à l'école primaire
}

\section{The schoolyard through the gender prism, a place of transformations in the responsibilities of teachers in elementary school \\ El patio de recreo bajo la perspectiva del género, lugar de transformación de las responsabilidades de los y las docentes en las escuelas primarias}

\section{Gaël Pasquier}

\section{Volume 41, numéro 1, 2015}

La responsabilité en éducation : transformations, ruptures et contradictions

Texte reçu le : 4 juin 2013, version finale reçue le : 24 février 2015, accepté le : 3 mars 2015

URI : https://id.erudit.org/iderudit/1031473ar

DOI : https://doi.org/10.7202/1031473ar

Aller au sommaire du numéro

Éditeur(s)

Revue des sciences de l'éducation

ISSN

0318-479X (imprimé)

1705-0065 (numérique)

Découvrir la revue

Citer cet article

Pasquier, G. (2015). La cour de récréation au prisme du genre, lieu de transformation des responsabilités des enseignant-e-s à l'école primaire. Revue des sciences de l'éducation, 41(1), 91-114. https://doi.org/10.7202/1031473ar

\section{Résumé de l'article}

En France, la cour de récréation des écoles primaires constitue un lieu où les enfants sont en grande partie livrés à eux-mêmes sous la surveillance des enseignant-e-s, dont la responsabilité consiste principalement à assurer la sécurité des élèves. Les politiques éducatives menées en faveur de l'égalité des filles et des garçons à l'école amènent cependant les maîtresses et les maîtres qui entendent se saisir de ces questions à envisager cet espace sous un jour nouveau, dans la mesure où ce fonctionnement participe activement à la reproduction des rapports sociaux de sexe traditionnels. Dans cet article, nous nous intéressons aux pratiques déclarées de ces professionnel-le-s, abordées à partir de vingt entretiens non-directifs. Nous y décrivons comment ces enseignant-e-s sensibles à la promotion de l'égalité sont amené-e-s à investir leur responsabilité dans et vis-à-vis de cet espace d'une dimension éducative qui les conduit à concevoir des formes d'interventions adaptées à la spécificité du lieu. 


\title{
La cour de récréation au prisme du genre, lieu de transformation des responsabilités des enseignant-e-s à l'école primaire
}

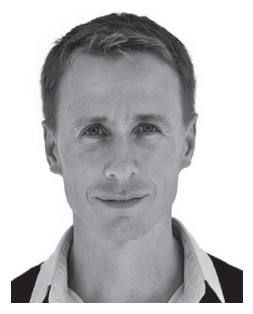

Gaël Pasquier

Maître de conférences Université Paris Est Créteil

\begin{abstract}
RÉSUMÉ - En France, la cour de récréation des écoles primaires constitue un lieu où les enfants sont en grande partie livrés à eux-mêmes sous la surveillance des enseignant-e-s, dont la responsabilité consiste principalement à assurer la sécurité des élèves. Les politiques éducatives menées en faveur de l'égalité des filles et des garçons à l'école amènent cependant les maîtresses et les maîtres qui entendent se saisir de ces questions à envisager cet espace sous un jour nouveau, dans la mesure où ce fonctionnement participe activement à la reproduction des rapports sociaux de sexe traditionnels. Dans cet article, nous nous intéressons aux pratiques déclarées de ces professionnel-le-s, abordées à partir de vingt entretiens non-directifs. Nous y décrivons comment ces enseignant-e-s sensibles à la promotion de l'égalité sont amené-e-s à investir leur responsabilité dans et vis-à-vis de cet espace d'une dimension éducative qui les conduit à concevoir des formes d'interventions adaptées à la spécificité du lieu.
\end{abstract}

MOTS CLÉS - égalité des sexes, école primaire, récréation, responsabilité des enseignant-e-s, genre.

\section{Introduction}

En France, la cour de récréation des écoles primaires constitue un lieu où les enfants sont en grande partie livrés à eux-mêmes sous la surveillance des enseignant-e-s. À l'inverse de ce qui se passe en classe, elle est conçue comme un espace de liberté où les contraintes imposées aux élèves se limitent pour l'essentiel à des questions de sécurité. Les modalités de surveillance sont définies en conseil des maîtres et des maîtresses sous la responsabilité du directeur ou de la directrice, afin de garantir la présence du nombre de personnes nécessaires 
à la sécurité des élèves en fonction des effectifs et de la configuration des lieux (ministère de l'Éducation nationale, 1997); et de fait, les enseignant-e-s sont bien souvent présent-e-s à tour de rôle dans la cour, alors que le nombre d'enfants est beaucoup plus important que durant les temps d'apprentissages institués. Plusieurs classes y sont en effet mises en présence pour une durée variant de 20 minutes en élémentaire, selon le cadre réglementaire de l'Éducation nationale, à 30 minutes dans certaines maternelles. La cour de récréation apparaît ainsi comme un lieu particulièrement propice à l'observation et à la compréhension des relations entre enfants (Delalande, 2001; Thorne, 1993). Elle l'est également pour analyser comment l'école participe à la reproduction des stéréotypes de sexe et des rapports sociaux de sexe traditionnels (Zaidman, 1996). Zaidman considère par exemple que l'absence d'investissement éducatif de cet espace par les maîtresses et maîtres d'école maternelle et élémentaire contribue à en faire un lieu d'expression et d'apprentissage de la domination masculine. Les élèves y apportent les occupations et les valeurs apprises dans et en dehors de l'école, et les réinvestissent dans leurs relations selon des modalités qui leur sont propres (Gayet, 2003; Rayou, 1999).

Or, en France, l'égalité des sexes constitue un objectif affiché par l'Éducation nationale depuis les années 1980 (Torsat, 1999), et plus particulièrement depuis l'an 2000, où a été signée la Convention interministérielle pour l'égalité entre les filles et les garçons, les femmes et les hommes, dans le système éducatif, réactualisée en 2006 et 2013. Comme l'ont encore montré, en 2013, la polémique autour des définitions considérées comme sexistes du Dictionnaire des écoliers de France, conçu à l'initiative du ministère de l'Éducation nationale, de la jeunesse et de la vie associative en 2011 (Pasquier, 2013a), ainsi que le rapport de l'Inspection générale de l'Éducation nationale (2013) consacré à L'égalité entre filles et garçons dans les écoles et les établissements, ces textes sont bien souvent peu appliqués. Pour autant, certain-e-s enseignant-e-s entendent travailler avec leurs élèves sur ces questions (Pasquier, 2010, 2013a, 2013b, 2013c, 2014a).

C'est à leurs pratiques déclarées que nous nous intéressons dans cet article. Nous présentons une partie des résultats d'une recherche réalisée à partir d'entretiens nondirectifs avec des professeur-e-s des écoles qui disent prendre en compte avec leurs élèves les questions relatives à l'égalité des sexes ou des sexualités. Notre question de recherche était la suivante: Que disent faire ces enseignant-e-s dans leur classe et dans leur école pour favoriser des rapports sociaux plus égalitaires entre filles et garçons, lutter contre les phénomènes d'homophobie et l'hétérosexisme? Cet article ne traitera ici que d'une partie du matériel recueilli en se centrant sur ce qui, dans leur discours, a trait à la cour de récréation et à l'égalité des sexes. Au regard des recherches existantes, il semble en effet judicieux de s'intéresser à la manière dont ils envisagent ce lieu et tentent de modifier les équilibres qui s'y établissent entre filles et garçons, tant sur le plan de l'occupation de l'espace que dans le choix des jeux. Nous faisons l'hypothèse que cette préoc- 
cupation nouvelle, l'égalité des sexes, amène ces enseignant-e-s d'école primaire à modifier les formes d'intervention qu'ils mettent en œuvre et la représentation qu'ils se font de leur mission éducative dans la cour de récréation. Ce faisant, nous cherchons à comprendre comment ils sont amenés à appréhender différemment leur responsabilité vis-à-vis de cet espace, habituellement envisagé sous un angle essentiellement sécuritaire.

Après avoir exposé le contexte théorique et la méthodologie utilisés pour cette recherche, nous présenterons les descriptions que les enseignant-e-s rencontré-e-s font de leur action dans et vis-à-vis de la cour de récréation avant de questionner ces résultats à l'aune du concept de responsabilité.

\section{Contexte théorique}

Il sera ici question de la cour de récréation comme lieu de désengagement des enseignant-e-s dans un contexte de reproduction des inégalités entre les sexes. Bien que l'égalité des sexes figure parmi les objectifs assignés à l'école mixte et républicaine en France, force est de constater que celle-ci ne réussit pas à traiter de la même manière les filles et les garçons (Duru-Bellat, 1995, 2010; Mosconi, 1989; Zaidman, 1996). Les pratiques enseignantes, les savoirs enseignés comme les supports utilisés véhiculent, bien souvent, des représentations inégalitaires et stéréotypées des femmes et des hommes, participant passivement et activement au fonctionnement du système de genre, entendu comme un système de normes de sexe qui légitime les inégalités entre les hommes et les femmes en les naturalisant (Marro, 2010). Ces processus sont particulièrement visibles dans la cour de récréation, où les relations entre les sexes semblent répondre à un principe d'évitement.

\subsection{La cour de récréation: un espace ségrégé sous le regard des adultes?}

En 1996, Zaidman a consacré une recherche à la mixité à l'école primaire en France, dont l'objectif était de mieux comprendre la manière dont certaines représentations et certains savoirs de sens communs concernant la différence des sexes informent le fonctionnement des écoles et la manière dont les enseignant-e-s interagissent avec leurs élèves. À partir d'une méthodologie mixte combinant des temps d'observations et des entretiens avec des maîtres et maîtresses travaillant dans des équipes mixtes, cette chercheuse a remarqué que la ségrégation spontanée des sexes s'associe généralement à un choix d'activités différentes et exclusives: le football et les jeux de course poursuite pour les garçons; les discussions, l'élastique ou la corde à sauter pour les filles (Zaidman, 1996, p. 55). À son tour, cette répartition n'est pas sans conséquences sur l'occupation de l'espace: les uns, nomades, l'investissent intégralement; les autres, plus sédentaires, doivent tenir compte des jeux des premiers pour choisir l'emplacement des leurs. Ces derniers ne risquent pas moins d'être envahis à tout instant en fonction des trajectoires de poursuite ou de balles. 
Les catégories de sexe ne sont toutefois pas toujours homogènes: Court (2010), ainsi que Joannin et Mennesson (2014) ont mis en évidence, dans le cadre de leurs observations en école élémentaire, la diversité et la variabilité selon les contextes des formes de masculinité et de féminité, en lien avec les caractéristiques sociales, scolaires et sportives des enfants. Par ailleurs, les processus d'évitement ne doivent pas masquer qu'il existe également des relations entre les filles et les garçons. À partir d'observations participantes menées dans deux écoles élémentaires étatsuniennes, en classe, en cours de récréation et dans d'autres espaces de la vie quotidienne des enfants à l'école (salles de restauration, couloirs...), Thorne (1993) s'est ainsi particulièrement intéressée à la question des frontières entre les groupes de sexe. Pour dénaturaliser ces groupes et rendre visibles les processus sociaux de construction des rôles et des normes de sexes, Thorne (1993, p. 65-66) a notamment repris la notion de borderwork ou travail des frontières pour désigner l'ensemble des interactions entre enfants qui produisent, rappellent et renforcent les limites entre les groupes de sexe et le rapport hiérarchique existant entre filles et garçons. Elle distingue étonnamment ces relations de celles auxquelles elle confère une dimension hétérosexuelle, alors que ces dernières pourraient sans doute constituer, dans bien des exemples décrits, une sous-section des premières. La chercheuse repère enfin des situations de transgression de ces frontières par des enfants isolés qui participent aux jeux de groupes composés d'enfants de l'autre sexe, ou des situations de jeux, en général organisées par des adultes, où filles et garçons jouent ensemble sans que les normes de distinction et de hiérarchisation des sexes ne semblent être à l'œuvre.

Parmi les relations de frontières, outre les tentatives de perturbation des jeux des filles par les garçons, ou inversement, il importe de mentionner certains jeux de poursuite. Zaidman (1996, p. 60), qui s'inscrit dans la lignée des travaux de Thorne (1993), précise que de tels jeux semblent jouir d'un grand prestige auprès des enseignant-e-s. Bien qu'ils mettent en général clairement en scène la poursuite des filles par les garçons et donc un rapport de force informé par la domination masculine, ces jeux marquent, en effet, des temps importants de la vie collective où les adultes suspendent leurs discussions pour observer ce qui se donne à voir comme un grand moment de partage entre enfants des deux sexes.

Ces observations ont été confirmées par des recherches ultérieures sur la cour de récréation comme celles de Delalande (2001) et de Ruel (2009) menées toutes deux dans une perspective anthropologique. Ces chercheuses combinent des observations et des entretiens d'enfants, avec pour objectif de mieux comprendre le fonctionnement de ce lieu spécifique pour la première, et celui de décrire les processus d'appropriation des normes du masculin et du féminin dans la construction des identités sexuées pour la seconde. De telles recherches tendent néanmoins, bien souvent, à occulter la relation de pouvoir mise en place entre les deux sexes, pourtant soulignée avant Delalande et Ruel par Thorne (1993) et Zaidman (1996), pour ne s'intéresser qu'aux processus de différenciation. En effet, 
si les filles et les garçons font bien, sous couvert d'autonomie et de liberté, des apprentissages différents, cette organisation de l'espace et des jeux favorise également la construction de rapports asymétriques et de domination entre les deux sexes: alors que les uns occupent la majeure partie des lieux et se construisent dans la rivalité, le corps à corps, la confrontation, les autres font l'expérience de la soumission et comprennent leur moindre importance par la superficie inférieure qui leur est accordée; superficie qui n'est pas sans conséquence, par ailleurs, pour leurs apprentissages moteurs.

\subsection{Quelle responsabilité enseignante dans cet espace?}

La position de retrait des maîtres et des maîtresses ne suffit cependant pas à expliquer pourquoi l'école favorise le développement des inégalités entre les sexes. Les recherches sur la mixité montrent en effet que les enseignant-e-s encouragent également à leur insu la domination des garçons sur les filles dans la gestion de leur classe (Duru-Bellat, 1995, 2010; Jarlégan, 1999; Mosconi, 1989; Pétrovic, 2004; Zaidman, 1996). La promotion de relations égalitaires entre les filles et les garçons interroge donc pleinement la manière dont chacun conçoit son rôle et sa responsabilité dans la cour de récréation. Delalande $(2005$, p. 27) remarque ainsi que les enseignant-e-s évoquent avant tout les questions de surveillance, cherchant prioritairement à identifier dans la cour un lieu qui leur permettra d'adopter un regard panoptique. Ce sont les recoins, les obstacles limitant leur champ de vision, qui les inquiètent au premier chef, car ils les empêchent de voir les comportements turbulents, les violences ou les accidents. Pause pour les enfants, la récréation est également implicitement considérée comme un temps de repos pour les maîtres et des maîtresses, même si celles-ci et ceux-ci restent vigilants-e-s afin d'éviter tout débordement. Elles et ils en profitent pour discuter entre eux, et préfèrent bien souvent renvoyer les enfants à la nécessité de régler eux-mêmes leurs problèmes et les conflits plutôt que de solliciter l'intervention d'un adulte (Delalande, 2005, p. 33; Zaidman, 1996, p. 65). Ce choix n'est pas sans conséquences: s'il encourage les enfants à se montrer davantage autonomes et à négocier entre eux pour arriver à leurs fins, il laisse aussi se manifester la loi du plus fort ou, occasionnellement, de la plus forte. La responsabilité des maîtres et des maîtresses durant ces temps de détente est donc à entendre dans un sens restreint, puisqu'elle concerne essentiellement la sécurité des corps et la possibilité de protéger ces derniers de toute blessure et agression. À l'inverse de ce qui se passe habituellement en classe, la responsabilité ne revêt pas à proprement parler de dimension éducative, puisqu'aucune finalité pédagogique n’y est associée.

Dans le cadre de la cour de récréation, l'exigence d'égalité des sexes semble donc devoir remettre en cause cette conception et transformer la manière dont les enseignant-e-s envisagent leur fonction. Responsables au sens courant du terme, puisqu'ils doivent garantir la sécurité de leurs élèves et sont redevables de leurs actes dans le cas où celle-ci serait compromise en raison de leur négligence, 
les enseignant-e-s le deviennent également dans un sens moral et éthique, puisqu'ils et elles doivent promouvoir l'une des valeurs essentielles aux démocraties occidentales contemporaines: l'égalité des sexes. Celle-ci étant posée comme un but à atteindre, les responsabilités afférentes et les actions qu'elles conditionnent trouvent leur justification dans la sphère d'influence du pouvoir confié au personnel enseignant (Jonas, 1990/1979, p. 182). Laisser se reproduire consciemment, au sein de l'enceinte scolaire, les inégalités entre les filles et les garçons par un refus d'agir s'apparente ainsi à un acte d'irresponsabilité, même si paradoxalement celui-ci n'est caractérisé par aucune action précise (Jonas, 1990/1979, p. 184-186). Cette passivité porte en effet préjudice à la fois aux élèves (filles ou garçons) infériorisés par la domination masculine, mais aussi à ce que le législateur définit comme un avenir souhaitable et susceptible d'être favorisé par certaines formes d'éducation.

L'objectif de cet article n'est toutefois pas d'examiner les questions que posent la transmission imposée de valeurs dans le cadre de l'école républicaine (Pasquier, 2010, p. 69-70); ces questions s'insèrent en effet, dans des problématiques attachées à l'éducation à la citoyenneté en général au sein de laquelle s'intègre en partie, et en partie seulement, l'éducation à l'égalité des sexes (Pasquier, 2013a). Nous entendons étudier la manière dont des enseignant-e-s engagé-e-s dans un travail en faveur de l'égalité des sexes avec leurs élèves disent agir dans la cour de récréation. Si ces nouvelles responsabilités, prises en charge par ces professeur-e-s des écoles dans cet espace, en lien avec la promotion de l'égalité des filles et des garçons, s'apparentent à celles qui leur sont confiées dans les apprentissages d'éducation civique, elles s'en distinguent également par les moyens d'actions qui s'offrent à eux. Leur marge de manœuvre apparaît en effet limitée par la spécificité même du lieu, dans la mesure où le fonctionnement de celui-ci n'est pas censé se calquer sur celui des lieux d'apprentissages ordinaires. Le temps de récréation doit demeurer un moment de détente et de liberté, une parenthèse dans l'emploi du temps des élèves, afin de leur permettre, lors de leur retour en classe, de retrouver une capacité de réflexion et de concentration optimale.

L'ensemble de ces paramètres laisse donc supposer que les enseignant-e-s préoccupé-e-s d'égalité des sexes doivent imaginer une manière originale d'exercer leurs prérogatives dans un endroit où une partie du contrôle de l'espace change de main et passe des adultes aux enfants. Ils ne sont donc pas uniquement investis de nouvelles responsabilités, au sens où ils doivent amener leurs élèves à appliquer les principes de l'égalité entre les filles et les garçons comme le leur demandent les Programmes d'enseignement de l'école primaire de 2008 (ministère de l'Éducation nationale, 2008, p. 29), cela tout en respectant, en tant que fonctionnaires de l'État, une nécessaire neutralité. Ils doivent en effet trouver des formes d'intervention qui tiennent compte de la fonction du lieu et du laps de temps qui lui est associé, fonction qui a priori ne leur permet pas de recourir à des formes traditionnelles d'enseignement ni d'agir comme ils le font habituelle- 
ment dans leur salle de classe. C'est la méthode utilisée pour avoir accès à ces différentes informations que nous allons maintenant présenter.

\section{Méthodologie}

Cet article s'inscrit dans une recherche visant à mieux connaître les pratiques des enseignant-e-s travaillant avec leurs élèves sur les questions relatives à l'égalité des sexes et des sexualités et leurs logiques d'action. Elle a été réalisée à partir de vingt entretiens nondirectifs, d'une durée moyenne d'une heure.

\subsection{Sujets}

L'échantillon des personnes rencontrées est diversifié sur le plan du sexe (15 femmes, cinq hommes), de l'âge, de l'ancienneté dans la profession (du stagiaire à l'Institut universitaire de formation des maîtres, aujourd'hui, École supérieure du professorat et de l'éducation, à la conseillère pédagogique récemment retraitée), du niveau d'enseignement (maternelle, élémentaire) et du lieu d'activité (Paris et sa banlieue, des zones urbaines ou rurales de province). Il n'ambitionne toutefois pas d'être représentatif, mais regroupe un ensemble d'informateurs privilégiés sur les formes que peut prendre une pédagogie antisexiste qui n'entend pas établir un lien entre le sexe d'un individu et un rôle à jouer dans la société.

\subsection{Instrumentation}

La question de départ des entretiens était la suivante: Vous travaillez sur l'égalité des sexes ou des sexualités avec vos élèves, je souhaiterais que vous m'en parliez: ce que vous faites, les réactions de vos élèves, des collègues, des parents, ce qui vous semble important. À partir de ce qui avait justifié la rencontre, cette phrase laissait entrevoir différentes entrées possibles: l'égalité des sexes et des sexualités bien évidemment et les conséquences possibles, que celles-ci soient considérées comme positives ou négatives, de l'introduction de cette préoccupation dans un champ professionnel qui ne s'en soucie habituellement pas ou peu.

Le choix d'une question aussi large se justifie par la multiplicité des moments et des disciplines susceptibles d'être concernés par les questions de genre, pour des professionnels qui se caractérisent par leur polyvalence. Par ailleurs, nous ne disposions pas d'informations précises sur les connaissances que ces enseignantse-s avaient des mécanismes de reproduction, par l'école, du système de genre, en dehors de leur intérêt professionnel et personnel pour l'égalité des sexes ou la lutte contre l'homophobie. Il nous a donc semblé judicieux de ne pas brider leur parole à partir de questions trop contraignantes sur des points particuliers (certaines disciplines scolaires, la gestion de la classe ou de lieux particuliers de l'école).

Aucune question précise n'a été posée sur la cour de récréation. Il en a été à chaque fois question à l'initiative de l'enseignant-e rencontré-e, et souvent dans la première moitié de l'entretien. Cet espace de l'école a donné lieu à des relances 
sous forme de reformulations de ce que la personne interrogée avait précédemment dit lors des six entretiens. Ces reformulations étaient destinées à amener ces enseignant-e-s à préciser leur pensée ou un récit.

\subsection{Déroulement}

Au départ difficiles à identifier, les enseignant-e-s que nous avons rencontré-e-s l'ont été, pour la plupart d'entre eux, par voie associative et syndicale de juin 2005 à juin 2012. Les rencontres ont eu lieu au fur et à mesure des identifications. Quatre entretiens ont été réalisés en juin 2005 ou durant l'année scolaire 20052006 dans le cadre d'un mémoire de master qui a posé les bases d'un travail de thèse. Douze entretiens ont été réalisés durant les années scolaires 2007-2008 et 2008-2009; quatre autres, menés en 2010 et en 2012, ont été ajoutés au corpus initial.

Cette période relativement importante ne semble toutefois pas à même de biaiser les résultats, car ceux-ci sont antérieurs aux polémiques déclenchées en France par la volonté affichée du ministère de l'Éducation nationale d'expérimenter dans des classes d'école primaire des séances de questionnement contre les stéréotypes de sexe. Les entretiens ont donc eu lieu sur une période où les changements en matière de lutte contre les discriminations sexistes et homophobes ont été peu significatifs dans la politique éducative menée par l'institution.

\subsection{Méthode d'analyse des données}

Les entretiens ont tous été enregistrés avec un dictaphone numérique, avec l'accord des personnes interrogées, puis intégralement retranscrits. Le contenu et la structure des données ont été analysées thématiquement à partir des grands ensembles qui se sont dégagés des entretiens et faisaient bien souvent échos aux recherches sur la mixité scolaire: 1) les savoirs disciplinaires (la grammaire, la littérature, l'éducation physique et sportive, l'histoire, l'éducation à la sexualité et l'éducation civique), 2) le cadre scolaire (la récréation, la gestion des interactions verbales, le placement dans la classe et la question des insultes), puis 3) un domaine transversal plus composite au sein duquel figuraient des questions liées à la forme scolaire et à la légitimité, pour l'école laïque et obligatoire, d'aborder en son sein des questions ne faisant pas consensus. La méthode d'analyse retenue s'inscrit dans les cadres de l'analyse critique de discours définis par Rogers (2011) et Rogers et ses collaboratrices (Rogers, Malancharuvil-Berkes, Mosley, Hui et Joseph, 2005; Rogers et Schaenen, 2013).

Bien qu'il soit nécessaire de supposer un écart entre le discours de ces enseignant-e-s et leurs pratiques effectives (Keddie, 1971; Thorel-Hallez, 2011) dans la mesure où la situation d'entretien conduit à une reconstruction de l'expérience vécue, la méthodologie choisie permet de combiner des informations factuelles avec la prise en compte d'éléments subjectifs qui renseignent sur l'intentionnalité éducative des personnes rencontrées et la façon dont elles perçoivent leur action. 
La question de départ, dans la mesure où elle les incitait à rendre compte de situations mises en œuvre avec leurs élèves sur un sujet encore peu pris en compte, les a conduits, parfois en fonction des réactions de leurs différent-e-s interlocuteur-rice-s dans leur environnement professionnel ou personnel, à faire part de leur propre questionnement sur l'égalité des sexes ou des sexualités, de leur cheminement et de leurs représentations. Dans cette recherche, nous ne visons donc pas à généraliser des analyses à partir de quelques individus, mais nous entendons proposer des pistes de réflexions visant à mieux comprendre les formes et la spécificité d'un enseignement qui tente d'enrayer la perpétuation de certaines formes de domination.

Il importe enfin de noter que la question des insultes homophobes a souvent été évoquée en lien avec la cour de récréation. Nous avons fait le choix de l'analyser séparément (Pasquier, 2014b) et de nous concentrer, dans cet article, sur les éléments concernant l'égalité des sexes.

\subsection{Considérations éthiques}

Les personnes ont été jointes par courriel. Le message qui leur a été envoyé leur indiquait l'objet de la recherche (les pratiques enseignantes en faveur de l'égalité des sexes ou des sexualités à l'école primaire), les informait que l'entretien serait enregistré et que des données recueillies seraient rendues anonymes, tous les noms, prénoms et les noms de lieux devant être modifiés. Un bref échange téléphonique destiné à préciser les modalités pratiques de la rencontre a eu lieu avant certains entretiens. Les entretiens se sont déroulés en cours d'année scolaire, sur le lieu de travail des personnes rencontrées, chez elles, ou dans un café. Enfin, nous avons proposé d'informer des résultats les enseignant-e-s qui le souhaitaient.

\section{Résultats}

Face aux inégalités entre les filles et les garçons et à leur séparation: quelle place et quel rôle pour les enseignant-e-s dans la cour de récréation? La méthodologie choisie n'incitait pas les personnes rencontrées à parler spécifiquement de la cour de récréation, mais les invitait simplement à évoquer leurs pratiques en lien avec l'égalité des sexes ou des sexualités. Il en est cependant question dans tous les entretiens, à l'exception d'un seul. Cette absence peut s'expliquer par le poste que l'enseignant concerné occupait: au moment de l'entretien, celui-ci travaillait alors en Section d'enseignement général adapté (SEGPA). Ce niveau de classe, où enseignent des professeur-e-s des écoles, se trouve localisé dans les collèges; or dans ces établissements, la surveillance de la cour n'est pas prise en charge par les enseignant-e-s, mais confiée à un personnel spécifique. La fréquence des propos concernant la récréation laisse donc entrevoir l'importance que celle-ci revêt aux yeux des personnes rencontrées dans le cadre de leur projet éducatif et les questionnements qu'elle suscite. Ce sont les extraits de leur discours en lien avec cet espace et ce moment que nous allons maintenant analyser. 


\subsection{La redéfinition de la place de l'adulte dans la cour de récréation}

Pour l'essentiel, les enseignant-e-s rencontré-e-s partagent les analyses de Zaidman (1996) sur la cour de récréation, qu'ils en aient connaissance ou qu'ils fassent spontanément les mêmes constats. Bien que cela ne concerne que quelques personnes interrogées, c'est donc tout d'abord la place de l'adulte que certains entretiens tentent de redéfinir. Si les propos de certain-e-s enseignant-e-s rappellent en effet clairement les écrits de Delalande (2001) et de Zaidman (1996), Quand on est de surveillance, on regarde, on surveille qu'il n'y ait pas d'incident (Béatrice), d'autres remarquent que cette attitude est aussi une manière de négocier en sous-main une certaine tranquillité: J'exagère peut-être, mais je me dis que ça arrange tout le monde; au moins pendant que ceux-là jouent au foot, ils n'embêtent personne; c'est une façon d'acheter la paix sociale; et là encore, on met les garçons en position haute parce que de toute façon, les filles, elles font pas chier [sic], alors c'est pas la peine de s'en occuper dans la cour; les garçons, si on les fait pas jouer au foot, il vont peut-être embêter les petits (Nathalie).

C'est donc en premier lieu l'investissement des enseignant-e-s dans cet espace que certaines des personnes interrogées souhaitent modifier à partir de leur propre expérience afin de le doter d'un véritable projet éducatif. Deux enseignantes choisissent ainsi de prendre une place active dans la cour pour initier une plus grande mixité dans les activités des élèves et encourager les filles qui le souhaitent à s'intégrer dans des jeux dont elles sont bien souvent exclues: Je joue avec les garçons pour leur montrer qu'une fille est capable de jouer au foot, et c'est vrai que les enfants aiment ça; ils viennent me chercher, même quand je ne suis pas de service, mais du coup, quand je me suis mise à jouer, les filles m'ont suivie (Samia). Ces enseignantes sont conscientes d'incarner un modèle en tant que femmes aux yeux de leurs élèves. Leur présence provoque par ailleurs un effet d'entraînement et d'adhésion d'autant plus fort qu'il joue un rôle sécurisant et participe à la pacification et à la régulation du jeu: Au départ, j'avais deux filles qui se mêlaient à moi et maintenant, j'en ai une dizaine; et elles y vont! (Samia). Cette situation est d'autant plus enthousiasmante et appréciée des enfants, filles et garçons, qu'ils n'y sont pas habitués, et qu'elle leur permet d'établir des relations de complicité avec leur enseignante dans un cadre moins formel que celui de la classe.

Ces enseignantes continuent toutefois à s'inscrire dans une perspective d'apprentissage: Ce que j'ai remarqué, c'est que les récrés où j'étais pas là, c'était assez cloisonné; et quand j'étais avec eux, moi, je joue avec eux dans la cour en général, je reste pas trop avec mes collègues; je jouais à la corde, j’organisais le jeu et du coup, c'était mixte; on rigolait et il y avait une belle émulation et une envie d'apprendre la corde pour les garçons; et puis j'allais sur le terrain de foot et du coup, j'amenais les quelques filles qui voulaient jouer, mais qui n'osaient pas dans mon équipe; mais c'est vraiment un travail (Camille). Un tel engagement est en effet, comme le remarque Camille, coûteux en énergie et sans doute difficile à mettre en place à chaque récréation, ce qui explique les réticences rencontrées 
par d'autres personnes interrogées lorsqu'elles ont proposé, sans succès, à leurs collègues d'organiser des jeux facultatifs durant ce temps. Cet engagement peut également nécessiter un nombre plus important d'adultes dans la cour, dans la mesure où il doit pouvoir être concilié avec les tâches de surveillance. Il permet cependant d'initier d'autres équilibres, de susciter certaines aspirations chez les élèves qui sont autant de portes vers l'égalité des sexes. Il s'agit, par l'engagement des adultes, d'ouvrir des possibles pour les filles et les garçons en leur permettant d'expérimenter des activités qu'ils ne se seraient pas nécessairement autorisés à pratiquer.

\subsection{L'établissement de liens avec les apprentissages d'éducation physique et sportive}

En remettant en question les stéréotypes de sexe, ces enseignantes cherchent aussi à encourager leurs élèves à développer les mêmes habiletés motrices: J'ai commencé à sauter à la corde, donc y'avait des gamins qui venaient me voir et des garçons qui disaient: "waouh»; et puis: "quand même, c'est un truc de filles"; justement, je leur parlais de la boxe; "Est-ce que tu connais Tyson? Il fait de la corde à sauter tous les jours». Du coup, un peu intrigués, ils ont essayé; et là, je me suis dit que les garçons avaient deux pieds gauches (Camille). Ce qui se passe dans la récréation n'est alors pas étranger à la vie de la classe (Joannin et Mennesson, 2014), notamment aux séances d'éducation physique et sportive. Ce lien est particulièrement sensible dans les entretiens, puisque plusieurs enseignant-e-s passent spontanément d'un sujet à l'autre. Ils considèrent que, puisque garçons et filles ne font pas, de par leur socialisation différenciée, les mêmes apprentissages moteurs, certaines tentatives pour les inciter à jouer ensemble risquent d'échouer. Pire, elles peuvent faire passer les différences entre les sexes pour ce qu'elles ne sont pas, c'est-à-dire les naturaliser (Marro 2010) au lieu d'inviter à les déconstruire: [les filles qui jouent au football] en prennent tellement ou alors on leur donne pas le ballon parce qu'elles savent pas tirer, parce qu'elles tirent un peu moins bien; c'est un peu normal, puisqu'elles y jouent moins que les garçons, mais du coup, les garçons prennent ça comme étant quelque chose de naturel, donc après, elles laissent tomber (Stéphanie). Selon ces enseignant-e-s, ici, la seule incitation à jouer ensemble ne suffit donc pas et ne saurait être productrice d'égalité, à moins que d'autres actions soient engagées, la mixité des groupes n'étant pas synonyme d'égalité.

Bien que certains d'entre eux et certaines d'entre elles n'entendent pas agir directement en récréation et souhaitent laisser aux enfants une partie de la maîtrise de cet espace et de ce temps, ils supposent toutefois que travailler sur ces questions à d'autres moments permettra ensuite aux enfants de réinvestir leurs nouvelles compétences dans la cour: Dans ma classe, j'essaie de mettre en place des jeux de ballons et j'essaie de donner les moyens aux filles d'être capables de jouer au foot; si on leur apprend pas à jouer et à se servir d'un ballon, à le manipuler, à tirer, elles 
savent pas (Samia). Ce travail ne doit d'ailleurs pas être réalisé qu'en direction des filles: J'essaye de donner les moyens aux filles de jouer au ballon et puis, par exemple, on joue à la corde à sauter aussi bien pour les garçons que les filles (Samia). Surtout, au-delà du simple développement de compétences corporelles, il doit permettre d'amener progressivement les élèves à apprécier jouer ensemble. En effet, comme le note une enseignante, ce plaisir ne va pas de soi: Il y a une élève de CM1 [Cours moyen, première année: enfants de 9 à 10 ans], qui est très forte au foot, et les garçons ont du mal à supporter qu'une fille soit meilleure qu'eux, donc ils essaient indirectement de l'évincer du jeu (Delphine). Ce que constate aussi l'une de ses collègues en pointant au passage la différence qui existe à ses yeux entre ce qui se passe en classe, dans les enseignements, et en récréation qui, sous couvert de liberté laissée aux élèves, ouvre la porte à l'exclusion: Par contre, dans les moments de récréation, elles vont pas sur le terrain, donc je pense que c'est là justement où notre rôle est important; c'est qu'on garantit finalement cet accès aux activités de garçons pour les filles et vice versa; mais quand on les laisse entre eux, c'est compliqué pour les filles de se faire une place; j'en ai une dans ma classe qui a joué quelques parties et qui me disait "mais j'en ai marre, ils me passent jamais la balle»; dans les faits, c'est égalitaire, c'est ouvert: "Venez, prenez de la place sur le terrain»; mais en vérité, on vous la laisse pas, cette place (Camille).

Accepter de jouer ensemble et prendre plaisir à le faire peut ainsi constituer une véritable gageure et reste dans tous les cas un apprentissage qui nécessite du temps, de la réflexion et beaucoup de pratique. La vie de la classe peut alors permettre, par les contraintes qu'elle impose, de faire découvrir aux élèves d'autres relations possibles entre les filles et les garçons que l'évitement et la rivalité. Nombre d'enseignant-e-s concerné-e-s considèrent par ailleurs que, même s'il s'agit d'un travail de longue haleine, les gains sont réels. Ils le sont sur le plan individuel, car le fait de jouer au foot peut avoir une véritable fonction émancipatrice pour les filles: [l'une des élèves de l'école] joue au foot et c'est la reine du monde; du coup, d'autres filles se sont mises à jouer et ça continue; je ne sais pas s'il y a un rapport de cause à effet, mais une gamine qui était mal dans ses baskets, un peu obèse, joue maintenant au football et elle est super heureuse (Hélène). Ils le sont également sur le plan collectif, pour le climat de l'école et la qualité des relations entre élèves, lorsque l'ensemble de l'équipe enseignante s'y associe.

\subsection{L'instauration de nouvelles règles d'occupation de l'espace}

Le fonctionnement de la cour de récréation, s'il semble parfois s'inscrire dans le rythme immuable des saisons qui amène chaque année les mêmes jeux et perpétue des traditions enfantines (Ottavi, 2009), n'est donc pas pour autant soumis à une fatalité qui cantonnerait les adultes à une position de spectateurs, simples garants de la sécurité des enfants mais exclus de toute initiative. L'autonomie et la liberté relatives des enfants sont dépendantes d'un cadre qui permet aux enseignant-e-s d'agir sur la manière dont ceux-ci s'approprient et investissent l'espace. Pour les 
personnes rencontrées, il ne s'agit pas, en effet, de mettre en place en récréation, sous prétexte d'assurer l'égalité entre les filles et les garçons, un contrôle des élèves équivalent à celui qui peut exister en classe. En revanche, elles entendent travailler sur les règles mises en place et leur propre investissement, afin de limiter autant que possible la répartition des élèves dans l'espace en fonction des catégories de sexe et les manifestations de domination des uns sur les autres. En effet, la cour n'est pas un espace vierge dans lequel les enfants projetteraient leurs jeux en dehors de toute contrainte: Est-ce que c'est les jeux qu'on leur impulse? Je vois bien dans ma cour, il y a deux marelles, deux terrains de foot et un terrain de basket au milieu, donc forcément, ça se fait de soi (Julie). Même si les élèves gardent toujours la possibilité de détourner son utilisation, le lieu induit un fonctionnement et des activités.

Toutefois, la présence de peintures au sol pour délimiter des aires de jeux ne constitue pas nécessairement un obstacle à l'égalité, bien au contraire. Car, comme le remarque Zaidman (1996, p. 56), la présence de séparations physiques entre les espaces de jeux canalise les activités des garçons, qui sans elles, tendent à s'étendre à l'ensemble de la cour, même lorsque certains espaces sont déjà occupés par d'autres. L'opportunité d'aménager la cour de manière à rendre possible la coexistence d'activités diversifiées constitue donc un atout non négligeable, mais qui peut considérablement varier selon la superficie du lieu: Il y a plusieurs paramètres: la taille, les différents lieux; si c'est une cour qui offre des petits coins, il y a moyen que chacun y trouve son compte, alors que s'il n'y a qu'un petit espace, il y a vite le monopole de certains ou de certaines (Danièle). Aussi, lorsqu'il n'y a qu'un seul terrain de football et que celui-ci est accaparé par des garçons qui acceptent de mauvaise grâce la présence des filles, certain-e-s enseignant-e-s proposent de réfléchir à la mise en place de règles de fonctionnement destinées à assurer l'égal accès des filles et garçons aux espaces les plus convoités: J'ai apporté ma pierre à l'édifice, car les filles se plaignaient parce qu'elles voulaient jouer au foot et qu'elles n'avaient pas assez de place; par la mise en place d'un planning, c'est tout simple; où jour par jour, on répartit la cour pour les filles et pour les garçons, que chacun ait au moins un espace équitable et que chacun puisse jouer au jeu qu'il souhaite (Aude).

Un tel dispositif peut cependant générer des tensions chez les garçons qui, pendant certaines récréations, n'ont pas accès au ballon, d'autant que les rares garçons qui choisissent tout de même d'aller jouer avec les filles peuvent être considérés comme des traîtres à leur groupe de pairs par leurs camarades du même sexe. Par exemple, Matthieu justifie d'avoir renoncé à cette alternance par l'intérêt de les récupérer, après la récréation, sans toute la haine qu'ils peuvent avoir les uns envers les autres quand ils n'ont pas eu le ballon ou dans un climat pas clivé avec les filles d'un côté, les garçons de l'autre, les filles en train de dire "Ouais, nous on a eu les ballons", les garçons "Ouais, vos jeux de meufs, ça me fait chier [sic]; les filles savent pas jouer au ballon, leurs jeux, c'est des jeux de gonzesses, quand 
on joue comme des filles, on se fait chier [sic], c'est tout mou» (Matthieu). Pour la remplacer, il impose le partage du ballon à chaque récréation: Au bout d'un moment, j'ai arrêté l'alternance filles/garçons, j'ai donné le ballon uniquement si les filles jouaient avec les garçons; j'essayais de les forcer à d'eux-mêmes se mixer et ça a fonctionné au bout d'un moment parce qu'ils savaient qu'[autrement] ils n'auraient pas le ballon; c'était à eux de se débrouiller, de décider des jeux qu'ils allaient faire et moi, je gardais un oil; je les avais prévenus qu'il fallait qu'ils arrivent à communiquer, à discuter (Matthieu). Il ne précise toutefois pas comment il a réussi à faire fonctionner cette solution dans une cour qui mélangeait plusieurs classes: chaque classe devait-elle gérer son propre ballon comme cela se passe dans certaines écoles? Toujours est-il qu'il concède au cours de l'entretien que son choix lui semble a posteriori trop radical vis-à-vis de certains garçons, et qu'il adopterait un système plus souple s'il se trouvait à nouveau confronté à une telle situation, tant leur frustration pouvait être grande.

Une autre possibilité, lorsque la taille de la cour le permet, consiste à proposer d'autres accessoires, même si les élèves détournent parfois leur usage de manière imprévue: J'ai fait acheter des cordes à sauter, des élastiques, des cerceaux, pour qu'il y ait aussi d'autres jeux; les cerceaux, ça a été un problème parce que souvent les garçons les balançaient d'un bout à l'autre de la cour et donc ils ont été détruits assez vite, et c'était pas terrible au niveau sécurité (Camille). D’autres personnes rencontrées tentent donc d'initier des jeux collectifs moins clivant, que le football, afin de constituer un répertoire connu des enfants, et que ces derniers pourront aisément mobiliser: J'avais proposé que sur une partie de la cour, on organise des jeux collectifs, mais qui soient pas marqués sexuellement, c'est-à-dire que ce soit pas le foot, que ce soit un épervier, la balle au camp, le béret; on avait trouvé des jeux, on essayait de définir les règles (Nathalie). Ce répertoire peut se constituer en référence aux cours d'éducation physique et sportive, et aux jeux collectifs qui y sont pratiqués. Il peut également, comme nous l'avons vu, être directement mis en place par l'intervention du maître ou de la maîtresse qui peut organiser certains jeux dans la cour. L'objectif est alors d'encourager les élèves à les poursuivre une fois l'adulte parti.

Par ailleurs, comme la cour de récréation est un espace dont l'organisation est en grande partie dévolue aux élèves, certain-e-s enseignant-e-s décident de les confronter directement à la question de son organisation afin de les responsabiliser sur son fonctionnement. La prise de conscience des inégalités permet ainsi de commencer un travail avec eux sur cette question: Y'a l'activité football évidemment; on a un créneau réservé pour chaque classe sur une récré de la semaine, donc je les laisse jouer la première semaine et puis après je leur demande un peu comment s'est passée la partie et j'amène l'idée: "Est-ce qu'il y avait des filles dans l'équipe?"; "Ah ben non"; donc on questionne un peu cette évidence-là parce qu'ils sont habitués depuis tout petits que les garçons occupent le terrain, que les filles soient dans un coin (Camille). Les cultures d'écoles et, plus précisément, 
l'existence d'institutions propres à encourager non seulement les débats et l'argumentation des unes et des autres mais aussi la prise de décision peuvent alors constituer des aides précieuses: Ici, il y a un conseil de classe avec les élèves, donc ils en parlent entre eux; on le fait d'abord en classe, donc forcément les délégués vont en parler ensuite, et il y a des choses qui se mettent en place; il y a quand même des avancées en récréation, on a eu en conseil de classe le souci que les filles n'avaient pas le droit de jouer au basket, parce que c'est vrai que la cour est grande, mais elle est investie par les garçons et quand il s'agit de jeux collectifs, les filles sont exclues, mais exclues vivement; donc là, c'est quand même un débat (Béatrice).

Ce sont alors les enfants qui réfléchissent eux-mêmes aux solutions à mettre en place, solutions qui sont d'autant mieux acceptées que les délégués de classes, filles et garçons, élues les ont défendues, votées et qu'ils peuvent en assurer la promotion auprès de leurs camarades: Il y a des choses qui se mettent en place, des délégués de classe qui doivent surveiller que les garçons n'évincent pas les filles et caetera (Béatrice). Ces enfants ont alors à cœur de mesurer la réussite de leur action: Au dernier conseil de classe, je n'yétais pas, mais j'ai vu sur le compte rendu que les délégués avaient dit que les garçons avaient mieux réagi au basket, que c'était les garçons qui avaient fait le plus d'efforts par rapport aux filles; alors je ne sais pas comment ils ont dit ça parce que c'est toujours des ressentis, mais c'est intéressant qu'on se pose des questions (Béatrice). Bien sûr, il est nécessaire, comme l'indique Béatrice, de considérer avec circonspection le ressenti des élèves: que peut bien vouloir dire que les garçons ont fait le plus d'efforts lorsque l'on sait que ce sont eux qui avaient jusqu'à maintenant le contrôle de l'espace de jeu et qu'ils peuvent également surévaluer leurs efforts aux yeux des autres et à leurs propres yeux? Pourtant, ces stratégies mises en place à l'échelle de la classe ou de l'école ne sont pas sans réussites: Les filles ont compris qu'elles avaient le droit d'investir toute la cour, donc je pense qu'il y a un progrès là-dessus (Béatrice).

\section{Discussion des résultats}

\subsection{Les formes d'extension de la responsabilité enseignante}

Si les entretiens réalisés témoignent des tentatives, essais et ajustements mis en place par les enseignant-e-s rencontré-e-s afin de promouvoir l'égalité des sexes dans la cour de récréation, force est de constater que les questions de responsabilité éducative ne s'y laissent lire qu'en filigrane. C'est avant tout leur engagement, direct ou indirect, vis-à-vis de cet espace, à la différence de ce qu'ils perçoivent chez les autres maîtres et maîtresses de leur école, qui permet en premier lieu de mesurer en quoi la situation les interpellent et pourquoi ils n'entendent pas s'en satisfaire. Car certaines enseignantes s'aperçoivent lorsqu'elles décident d'aborder cette question avec leurs collègues qu'ils sont extrêmement conscients du rapport de force qui existe dans la cour, mais décident volontairement de ne pas y toucher afin de ne pas avoir à en subir les conséquences: J'ai amené cette question au conseil des maîtres parce que, comme dans toutes les écoles, le foot prend beaucoup 
de place et les garçons aussi, et ça a été un tollé: "Ah mais non, on est bien tranquilles avec les garçons qui jouent au foot " (Camille). La recherche de dispositifs visant à modifier les rapports de domination à l'œuvre dans cet espace témoigne donc d'une prise de distance vis-à-vis de ces conceptions et de l'élargissement, par les personnes rencontrées, du périmètre d'action dévolu au personnel enseignant. Ces personnes entendent ainsi remettre en cause la manière dominante dont sont perçus les moments informels de la vie de l'école, où les élèves seraient comme soustraits à l'action des adultes (Thorne, 1993; Zaidman, 1996, p. 65).

Si certain-e-s professeur-e-s des écoles font le choix de s'investir directement et physiquement dans les jeux des enfants et leur organisation, la plupart d'entre eux préfèrent cependant réfléchir à la manière de laisser aux élèves une part de liberté dans ce temps réservé à la détente, sans pour autant se déresponsabiliser de la manière dont ceux-ci l'utilisent. Le lien entre la récréation et la classe, les institutions de l'école, constituent alors des recours qui permettent d'agir indirectement pour un rééquilibrage des relations entre les sexes. La récréation n'est donc pas considérée comme un moment à part où les responsabilités éducatives des maîtres et des maîtresses devraient demeurer en retrait. Elle est, au contraire, à l'instar de ce qui se passe en classe mais différemment, un lieu où ceux-ci se sentent pleinement responsables des relations que les élèves établissent entre eux, des compétences qu'ils ont acquises et manifestent, dans la mesure où celles-ci ne sont pas figées. Les enseignant-e-s ont en effet sur ces relations et ces compétences un pouvoir d'action en raison de la responsabilité contractuelle (Jonas, 1990/1979) qui leur a été confiée et qu'ils ont acceptée: sur l'ensemble du temps scolaire, les professeur-e-s sont susceptibles d'enseigner des connaissances, de transmettre des valeurs et de mettre en place les conditions nécessaires à l'acquisition de certaines compétences par les élèves. Dans ce lieu comme dans le reste de l'école, leur responsabilité n'est donc plus uniquement conçue par rapport au présent et à la sécurité physique et matérielle de leurs élèves, mais s'envisage également en référence à l'avenir. En effet, la séparation de l'espace et des activités en fonction du sexe de l'enfant peut avoir, comme l'indique une enseignante, des conséquences super graves sur la construction identitaire masculine ou féminine, et c'est pour ça qu'aujourd'hui, je m'investis beaucoup avec le sport parce que j'ai l'impression qu'en prenant cet espace physiquement, les filles prennent aussi de l'espace mental (Camille).

Il n'en reste pas moins que certaines solutions proposées par les enseignant-e-s rencontré-e-s perpétuent cette séparation des sexes et peuvent de ce fait paraître problématiques, comme celles qui consistent à confier un ballon spécifique au groupe de filles ou au groupe de garçons, ou à leur réserver alternativement le terrain de foot de la cour. Outre que ces propositions d'organisation comportent l'inconvénient de ne pas inciter les garçons et les filles à jouer ensemble, elles semblent impliquer que tous les garçons qui veulent jouer sur le terrain ont la possibilité de le faire lors du créneau réservé à leur sexe, ce qui n'est en réalité pas 
le cas. Joannin et Mennesson (2014, p. 165 et 177) ont en effet montré que certains garçons sont exclus du jeu par leurs pairs qui sélectionnent les coéquipiers auxquels ils font des passes, et finissent de ce fait par renoncer à y participer. Imposée par l'enseignant-e, la scission entre les filles et les garçons n'a par ailleurs pas forcément la même signification aux yeux des élèves que celle que ceux-ci opèrent spontanément: les filles et les garçons peuvent en effet considérer que si un adulte prend la peine de les séparer, c'est bien parce qu'ils sont fondamentalement différents. Néanmoins, malgré la pertinence de ces critiques, ces dispositifs permettent d'assurer l'accès de certaines élèves à des espaces qui leur sont habituellement interdits par l'usage établi.

Les solutions mises en place par les enseignant-e-s rencontré-e-s n'en montrent pas moins que la plupart d'entre eux, lorsqu'il s'agit de la cour de récréation, entendent exercer ces responsabilités de façon médiatisée. Seules deux enseignantes affirment organiser des jeux avec les enfants, ce qui amène à s'interroger sur les réticences des maîtres des deux sexes à s'investir directement dans cet espace. Le souci de se concéder un temps de pause en même temps qu'aux élèves n'est probablement pas négligeable, mais doit sans doute être associé à d'autres craintes comme celle d'outrepasser son rôle en optant pour un mode d'action qui pourrait s'apparenter à celui des animateurs et des animatrices chargé-e-s des enfants sur le temps périscolaire et ainsi de dévaluer sa profession. Les informations contenues dans les entretiens ne nous permettent toutefois pas d'étayer cette hypothèse, même s'il nous semble utile de la formuler en raison des hiérarchies implicites à l'œuvre dans les représentations communes concernant les modes d'accueil de l'enfance et leurs finalités. Les tentatives pour établir des liens entre la cour et la classe peuvent donc également être analysées comme un moyen implicite d'affirmer la spécificité de la position des enseignant-e-s dans un espace où d'autres formes d'action risqueraient de générer des confusions et une rupture trop grande avec la conception que les personnes interrogées ont de leur métier.

\subsection{Les liens entre la cour de récréation et la classe en question}

Il est alors utile de considérer la manière dont ce qui se passe en classe est mis à profit pour modifier les comportements dans la cour. Même si ces deux espaces fonctionnent selon des logiques différentes, leur clivage ne doit pas être exagéré. Ils sont tous deux investis par les mêmes acteurs, qui, s'ils s'engagent dans des interactions différentes, n'en restent pas moins porteurs de ce qui s'est passé auparavant. Aussi, comme le note Gayet (1998, p. 16), si l'enfant va parfois solliciter à l'école les représentations de son milieu familial en lien avec des expériences qu'il y a faites, il ne se défait pas non plus entièrement, lorsqu'il arrive en récréation, de ce qu'il a appris et vécu en classe, même s'il le met en jeu de manière différente (Delalande, 2005, p. 35).

C'est donc cette frontière que les professeur-e-s des écoles rencontré-e-s entendent questionner pour étendre leur influence, mais elle peut également leur 
sembler problématique dans la mesure où les mécanismes d'appropriation par les élèves des valeurs défendues en classe par l'enseignant-e leur restent bien souvent obscurs et complexes à analyser. Ils constatent ainsi, parfois avec surprise, que ce sur quoi ils ont travaillé, lorsqu'ils ont par exemple mené un projet particulier autour des stéréotypes de sexe attachés aux jouets, interrogé les rôles traditionnellement attribués aux garçons et aux filles dans la littérature de jeunesse ou encouragé la mise en place d'une réelle mixité dans tous les moments de la vie de leur classe, n'est pas sans conséquences sur les jeux des enfants: Alors après, ça fait un peu bizarre parce qu'il y a par exemple les CM1 B [Cours moyen, première année: enfants de 9 à 10 ans], où les filles et les garçons se mélangent (Julie); Ce qui a été acquis, c'est que les élèves de notre classe sont moins excités et plus fairplay (Delphine). La cour de récréation est alors un moment partagé avec d'autres classes qui permet souvent involontairement, par comparaison avec les élèves de ses collègues, de mesurer ou non les effets de son action.

Pourtant, quelques remarques montrent que certaines personnes interrogées peinent à percevoir comment leurs élèves tissent des liens entre la classe et la cour, et pourraient faire croire que cette question n'est pas toujours travaillée: Je ne sais pas comment ils arrivent à faire ce lien, parce que c'est vrai que là, je vois des progrès quand même assez rapides (Béatrice). Alors que l'extension de la responsabilité enseignante à la manière dont les élèves investissent la cour de récréation est perceptible dans les discours, la construction par les élèves d'une forme de continuité entre cet espace et la classe semble devoir rester mystérieuse. Ce ressenti, ici exprimé par Béatrice, paraît d'autant plus surprenant que cette même enseignante décrit à d'autres moments comment elle amène ses élèves à se saisir des conseils des délégués de classe pour porter une revendication d'égalité.

La transformation de la responsabilité des personnes rencontrées vis-à-vis de la récréation consiste en effet pour beaucoup à inscrire dans le temps de classe des préoccupations liées à ce qui se passe dans la cour. Les maîtres et les maîtresses ont alors recours à des disciplines instituées comme l'éducation physique et sportive ou à des dispositifs pédagogiques familiers des enseignant-e-s du primaire, à commencer par le débat réglé, qui permettent aux élèves de progressivement s'approprier les questions posées par l'adulte: Je me souviens que l'année dernière, on avait parlé de l'occupation de la cour par le jeu du football et, donc, du coup, les garçons se remettaient en cause; ça avait donné toute une discussion; les filles disaient: "Nous, on n'a pas le droit d'y jouer»; les garçons disaient : «Mais vous savez pas jouer»; les filles: «Oui, mais si on joue jamais, on saura pas jouer» (Nathalie). La discussion initiée par Nathalie vise clairement à interroger l'ensemble des élèves sur l'organisation de la cour et leurs habitudes. Les garçons considèrent en effet comme légitime d'occuper la majeure partie de l'espace, car leurs jeux nécessitent de la place, leur semblent plus importants que les jeux des filles qui ne les intéressent pas; ils n'éprouvent donc aucune gêne à perturber ceux-ci en les traversant (Ruel, 2009, p. 207). Leur réaction au cours de ce débat 
est d'ailleurs symptomatique de cette situation: jouer à leurs jeux et plus particulièrement au football nécessite un savoir qu'ils seraient les seuls à posséder. Les filles, de leur côté, formulent leur exclusion des jeux de ballons en terme de droit, ce que constate également à plusieurs reprises Delalande dans sa recherche (2001, p. 154 et 164).

La sociologue ne perçoit cependant, dans l'utilisation de ces termes, [on n'a pas le droit] que l'expression de la reprise à leur compte, par les enfants, d'une conception culturelle de la séparation des sexes transmise par les adultes (p. 164). La formulation explicite d'un tel interdit par un adulte nous semble, pour notre part, beaucoup moins improbable. Surtout, l'expression ne pas avoir le droit nous semble témoigner de l'intégration par les filles de la domination masculine, dimension occultée par la chercheuse, qui accole d'ailleurs au nom fille un diminutif qu'elle n'utilise pas lorsqu'il s'agit de nommer les garçons, puisqu'il n'est pas question dans son analyse de garçonnets, alors qu'elle parle de fillettes. Dans la scène rapportée par Nathalie, les filles ont donc très bien compris que, dans une telle situation, elles n'étaient pas en mesure de dire le droit parce qu'elles ne détenaient pas le pouvoir. Le glissement qui s'opère dans la discussion entre le fait d'avoir le droit de jouer au football et celui de savoir jouer au football est donc particulièrement intéressant, puisqu'il permet de réinscrire la situation dans une dynamique de changement et de mettre en évidence un facteur susceptible de la modifier: l'apprentissage. Et comme celui-ci ne peut avoir lieu que par l'expérimentation, un tel constat rend possible, voire nécessaire, l'intégration des filles aux jeux de ballons.

Ces discussions sont donc conçues par les enseignant-e-s comme des moments où des formes d'apprentissages scolaires connues des enfants entrent en résonnance avec ceux où filles et garçons gèrent leurs occupations de façon autonome. L'éducation civique n'est toutefois pas le seul moment où ce transfert est possible; certain-e-s professeur-e-s choisissent également de l'effectuer dans d'autres enseignements comme ceux consacrés à la littérature: Je vais travailler dans trois classes de Cycle 3 [Cours élémentaire, deuxième année, cours moyen 1 et 2: enfants de 8 à 11 ans] dans une école où la problématique, c'est qu'il y a beaucoup de bagarres dans la cour de récréation sur le thème "hein, elle est amoureuse; hein, il est amoureux, ninninnin "; et donc, j'ai proposé aux enseignantes de traiter la question par le biais de la littérature de jeunesse (Sandrine). Sandrine ne précise pas si elle compte expliquer à ses élèves les raisons de son choix thématique, mais elle escompte, pour le climat de l'école, un bénéfice de ce travail qui, en faisant écho aux préoccupations des enfants, les pousse à relier deux espaces qu'ils envisagent parfois de manière séparée. Cette enseignante fait le pari qu'en mettant des mots sur certaines situations problématiques, sans que celles-ci soient nommées directement ou sans qu'il y soit forcément fait référence, les enfants seront en mesure de les envisager plus sereinement. La littérature est ici bien autre chose qu'un simple objet d'apprentissage permettant l'étude de la langue ou de structures 
narratives, elle se trouve en prise directe avec la vie. La constitution d'une culture littéraire adaptée à l'âge des enfants vaut alors par la diversité des modèles qu'elle offre et parce qu'elle permet de dépasser des craintes, de nommer ou de retrouver l'expression de sensations et de sentiments éprouvés, d'élargir enfin sa sensibilité au monde et aux autres pour les aborder d'une manière décalée ou nouvelle, débarrassée de certaines angoisses et enrichie de l'expérience des autres, fût-elle fictive.

\section{Conclusion}

Les objectifs de la recherche dont une partie des résultats sont présentés dans cet article étaient de rendre compte des pratiques déclarées d'enseignant-e-s d'école primaire en faveur de l'égalité des sexes et des sexualités. Dans la mesure où il a été fréquemment et spontanément question de la gestion de la cour de récréation dans les entretiens réalisés, il a semblé pertinent de s'intéresser plus particulièrement au rapport que les personnes rencontrées entretiennent avec cet espace et aux modes d'action qu'elles disent y mettre en œuvre. Nous avons donc cherché, dans cet article, à déterminer dans quelle mesure le souci de promouvoir des rapports plus égalitaires entre filles et garçons modifiait la manière dont les enseignant-e-s d'école primaire percevaient leur responsabilité dans et vis-à-vis de la cour de récréation.

À les écouter, cette préoccupation n'est pas sans influence sur leurs représentations et leurs pratiques. Si la plupart préfèrent agir sur la cour de récréation de façon médiatisée, les enseignants des deux sexes l'investissent toutefois d'objectifs éducatifs qui en étaient auparavant majoritairement absents. Le désir de promouvoir des relations plus égalitaires entre les filles et les garçons, des conduites moins stéréotypées, les amène à envisager cet espace sous un jour nouveau qui, sans mettre de côté les questions de surveillance et la limitation des phénomènes violents ou des accidents, redéfinit plus ou moins profondément leur mission. Pour autant, bien que ce point n'ait été qu'indirectement abordé dans cet article, l'extension de leurs responsabilités n'est pas sans générer des tensions dans la vie de l'école. Si la récréation questionne la capacité des enfants à jouer ensemble, elle interroge également celle des adultes à travailler en équipe. Certain-e-s enseignant-e-s reconnaissent ainsi se sentir isolé-e-s et disent rencontrer des difficultés à convaincre leurs collègues du bien-fondé de leur approche. Pour leurs élèves, les habitudes prises avec les enfants des autres classes dans les jeux et l'occupation de l'espace se révèlent alors parfois des obstacles extrêmement puissants pour les valeurs que ces adultes tentent de promouvoir. La cour de récréation représente en effet un espace au carrefour de l'école mais aussi des apprentissages, révélateur non seulement du niveau d'appropriation par les élèves, de certains savoirs et certaines valeurs, mais aussi des tensions à l'œuvre dans les représentations que les enseignant-e-s du primaire ont de leur métier, entre éducation et instruction. Il serait ainsi particulièrement judicieux, en prolongement de cette 
recherche, de s'intéresser à des écoles regroupant plusieurs enseignant-e-s engagée-s dans un travail en faveur de l'égalité des sexes. L'objectif pourrait alors être, en combinant des temps d'observation et des entretiens, d'envisager comment s'effectue ou non le passage d'une conception de la responsabilité, certes élargie mais individuelle, à une responsabilité collective à l'ensemble de l'équipe des maîtres et maîtresses, conception de la responsabilité qui ne se limiterait pas à prévenir les seuls accidents.

\section{ENGLISH TITLE - The schoolyard through the gender prism, a place of transformations in the responsibilities of teachers in elementary school}

SUMMARY - In France, the schoolyard of the primary school is a place where children are largely left to themselves and under the supervision of teachers whose main responsibility is to ensure the safety of pupils. However, educational policies in favor of the equality between girls and boys in school lead teachers who intend to take up these issues to consider this area under a new light due to the fact that this behavior is actively involved in the reproduction of traditional gender relations. This article deals with the practices declared by these professionals on the basis of twenty non-directive talks. It describes how these teachers who are sensitive to the promotion of equality invest this place with an educational dimension which leads them to develop forms of intervention suited to this specific place.

KEY WORDS • gender, primary school, schoolyard, responsibility of teachers.

TítULO - El patio de recreo bajo la perspectiva del género, lugar de transformación de las responsabilidades de los y las docentes en las escuelas primarias

RESUMEN - En Francia, el patio de recreo de las escuelas primarias constituye un lugar donde los niños son en gran medida dejados en libertad, bajo la supervisión de docentes cuya responsabilidad consiste principalmente en velar por la seguridad de los niños. Sin embargo, las políticas educativas desarrolladas en favor de la igualdad entre niños y niñas en la escuela conducen a los y a las docentes sensibles a estas cuestiones a considerar este espacio bajo una nueva perspectiva, dado que este funcionamiento interviene activamente en la reproducción de las relaciones sociales tradicionales entre sexos. Este artículo se interesa por las prácticas declaradas de estos y estas profesionales, abordadas a través de veinte entrevistas no dirigidas. Se describe cómo estos y estas docentes sensibles a la promoción de la igualdad acaban ampliando sus responsabilidades en, y con respecto a, este espacio de una dimensión educativa que les lleva a desarrollar modos de intervención adaptados a la especificidad de este lugar.

PALABRAS CLAVE - igualdad de género, escuela primaria, recreo, responsabilidad de los y las docentes.

\section{Références}

Court, M. (2010). Corps de filles, corps de garçons: une construction sociale. Paris, France: Éditions La Dispute.

Delalande, J. (2001). La cour de récréation. Pour une anthropologie de l'enfance. Rennes, France: Presses universitaires de Rennes. 
Delalande, J. (2005). La cour d'école: un lieu commun remarquable. Recherches familiales, 1(2), 25-36.

Duru-Bellat, M. (1995). Filles et garçons à l'école. Note de synthèse. Deuxième partie: La construction scolaire des différences entre les sexes. Revue française de pédagogie, 110, 75-109.

Duru-Bellat, M. (2010). Ce que la mixité fait aux élèves. Revue de l'OFCE, 114, 197-212.

Gayet, D. (1998). École et socialisation. Le profil social des écoliers de 8 à 12 ans. Paris, France: L'Harmattan.

Gayet, D. (2003). L'élève, côté cour, côté classe. Paris, France: Institut national de recherche pédagogique.

Inspection générale de l'Éducation nationale (2013). L'égalité entre filles et garçons dans les écoles et les établissements. Rapport à Monsieur le ministre de l'Éducation nationale. Paris, France: ministère de l'Éducation nationale.

Jarlégan, A. (1999). La fabrication des différences: sexe et mathématiques à l'école élémentaire. (Thèse de doctorat non publiée). Université de Bourgogne, France.

Joannin, D. et Mennesson, C. (2014). Dans la cour de l'école. Pratiques sportives et modèles de masculinités. Cahiers du genre, 56(1), 161-184.

Jonas, H. (1990/1979). Le principe de responsabilité. Paris, France: Éditions Flammarion.

Keddie, N. (1971). Classroom Knowledge. In M. F. D. Young (ed.), Knowledge and control: new directions for the sociology of education (p. 133-160). London, United Kingdom: Collier-MacMillan.

Marro, C. (2010). Sexe, genre et rapports sociaux de sexe. Dans A. Olivier (dir.), Sexe, genre et travail social. Paris, France: L'Harmattan.

Ministère de l'Éducation nationale (1997). Circulaire du 18 septembre 1997 sur la responsabilité et la sécurité dans les écoles élémentaires et maternelles publiques. Paris, France: Ministère de l'Éducation nationale.

Ministère de l'Éducation nationale (2008). Horaires et programmes d'enseignement de l'école primaire. Bulletin officiel du ministère de l'Éducation nationale et du ministère de l'Enseignement supérieur et de la recherche, 3.

Ministère de l'Éducation nationale (2013). Convention interministérielle pour l'égalité entre les filles et les garçons, les femmes et les hommes dans le système éducatif. Paris, France: Ministère de l'Éducation nationale.

Ministère de l'Éducation nationale, de la jeunesse et de la vie associative (2011). Le Dictionnaire des écoliers de France, vol. 5-8 ans et vol. 8-11 ans. Paris, France: Larousse, SCÉRÉN (Services Culture Édition Ressources pour l'Éducation nationale), Centre national de documentation pédagogique - Centre régional de documentation pédagogique.

Mosconi, N. (1989). La mixité dans l'enseignement secondaire: un faux semblant? Paris, France: Presses universitaires de France.

Ottavi, D. (2009). «Ah! mais c’est un jeu d'été, ça!» La pérennité d’un entre-enfants étudiée au début du $\mathrm{xx}^{\mathrm{e}}$ siècle par Roger Cousinet. Dans J. Delalande (dir.), Des enfants entre eux. Des jeux, des règles, des secrets (p. 12-28). Paris, France: Éditions Autrement. 
Pasquier, G. (2010). Enseigner l'égalité des sexes. Nouvelles questions féministes, 29(2), 60-71. Lausanne, Suisse: Éditions Antipodes.

Pasquier, G. (2013a). L'enseignement de la langue française à l'école primaire à l'épreuve de la déconstruction du genre. Formation et pratiques d'enseignement en questions, $16,161-178$.

Pasquier, G. (2013b). Enseigner l'égalité des sexes à l'école primaire: des pratiques qui se cherchent. Dans C. Morin-Messabel (dir.), Filles/garçons. Questions de genre de la formation à l'enseignement (p. 281-300). Lyon, France: Presses universitaires de Lyon.

Pasquier, G. (2013c). Enseigner l'égalité des sexes : quelles pratiques pour quels contenus d'enseignement. Dans M. Estripeaut-Bourjac et N. Sembel (dir.), Femmes, travail, métiers de l'enseignement: rapports de genre et rapports de classe (p. 285-294). Rouen, France: Presses universitaires de Rouen et du Havre.

Pasquier, G. (2014a). Enseigner l'égalité des sexes par la littérature de jeunesse à l'école primaire: quelle place pour les garçons? Dans S. Ayral et Y. Raibaud (dir.), École, loisirs, sports, culture: la fabrique des garçons (p. 141-164). Bordeaux, France: Maison des sciences de l'homme d'Aquitaine.

Pasquier, G. (2014b). Des enseignant-e-s face aux insultes homophobes. Raisons éducatives, $18,195-217$.

Pétrovic, C. (2004). Filles et garçons en éducation: les recherches récentes. Deuxième partie. Carrefour de l'éducation, 18, 3-31.

Rayou, P. (1999). La grande école. Approche sociologique des compétences enfantines. Paris, France: Presses universitaires de France.

Rogers, R. (ed.) (2011). Introduction to critical discourse analysis in education ( $2^{\text {nd }}$ edition). New York, New York: Routledge.

Rogers, R. et Schaenen, I. (2013). Critical discourse analysis in literacy education : a review of the literature. Reading research quarterly, 49(1), 121-143.

Rogers, R., Malancharuvil-Berkes, E., Mosley, M., Hui, D., and Joseph, G. O. (2005). A critical review of critical discourse analysis. Review of research in education, 75(3), 365-416.

Ruel, S. (2009). La construction culturelle des sexes du point de vue des enfants. L'enfant, sujet actif dans le processus de socialisation et de formation des identités de genre (Thèse de doctorat non publiée). Université de Caen/Basse-Normandie, France.

Thorel-Hallez, S. (2011). De la mixité à la coéducation en danse contemporaine au collège. Analyse de l'activité d'enseignant-e-s d'éducation physique et sportive. Paris, France: L'Harmattan.

Thorne, B. (1993). Gender play. Girls and boys in school. New Brunswick, New Jersey: Rutgers University Press.

Torsat, D. (1999). Les politiques d'égalité à l'école entre les filles et les garçons, 1984-1999. Dans F. Vouillot (dir.), Filles et garçons à l'école: une égalité à construire (p. 39-43). Paris, France: Centre national de documentation pédagogique.

Zaidman, C. (1996). La mixité à l'école primaire. Paris, France: L'Harmattan. 
Monsieur Gaël Pasquier est Maître de conférences en sociologie à l'École Supérieure du Professorat et de l'Éducation, à l'Université Paris Est Créteil; il est également chercheur à l'Observatoire universitaire international éducation et prévention (OUIEP) et au Laboratoire interdisciplinaire de recherche sur les transformations des pratiques éducatives et des pratiques sociales (LIRTES).

\section{Correspondance}

gael.pasquier@u-pec.fr

\section{Contribution de l'auteur}

Gaël Pasquier: $100 \%$

Ce texte a été révisé par Geneviève Falaise.

Texte reçu le: 4 juin 2013

Version finale reçue le: 24 février 2015

Accepté le: 3 mars 2015 